\title{
When Sick Brain and Hopelessness Meet: Some Aspects of Suicidality in the Neurological Patient
}

\author{
Alessandra Costanza ${ }^{1,2, *}$, Andrea Amerio ${ }^{3,4,5}$, Andrea Aguglia ${ }^{3,4}$, Andrea Escelsior ${ }^{3,4}$, \\ Gianluca Serafini ${ }^{3,4}$, Isabella Berardelli ${ }^{6}$, Maurizio Pompili ${ }^{6}$ and Mario Amore ${ }^{3,4}$ \\ ${ }^{I}$ Department of Psychiatry, Faculty of Medicine, University of Geneva (UNIGE), Geneva, Switzerland; ${ }^{2}$ Department of \\ Psychiatry, ASO Santi Antonio e Biagio e Cesare Arrigo Hospital, Alessandria, Italy; ${ }^{3}$ Department of Neuroscience, \\ Rehabilitation, Ophthalmology, Genetics, Maternal and Child Health, Section of Psychiatry, University of Genoa, \\ Genoa, Italy; ${ }^{4}$ IRCCS Ospedale Policlinico San Martino, Genoa, Italy; ${ }^{5}$ Mood Disorders Program, Tufts Medical \\ Center, Boston, MA, USA; ${ }^{6}$ Department of Neurosciences, Mental Health and Sensory Organs, Suicide Prevention \\ Center, Sant'Andrea Hospital, Sapienza University of Rome, Rome, Italy
}

\begin{abstract}
Neurological diseases expose individuals to a higher risk of suicidal ideation and suicidal behavior, including completed suicides and suicide attempts. They also represent a paradigmatic arena to study the etiopathogenic mechanisms underlying suicidality because they are emblematic of the heterogeneity and complexity of mutual interrelationships characterizing this issue. On the one hand, neurological diseases imply strictly biological impairments that are postulated to be the basis of vulnerability to suicide or result in the need for treatments for which a suicidal risk has been hypothesized. On the other hand, they question some subjective experiences of neurological patients, up to near existential positions. Often, in fact, they are accompanied by severe hopelessness. The latter may originate in, particularly for the most severe neurological diseases, the absence of curative treatments, unpredictable disease progression that leads to acute relapses or chronicity, a decrease in autonomy or selfidentity, progressive social isolation, a sense of becoming useless, and perception of feeling stigmatized. This may ultimately cause a slip into experiencing an absurd condition. At the confluence of neurobiology and hopelessness, frequent psychiatric comorbidities may play a primary role. To conclude, neurological patients require special attention from clinicians in form of openly verbalizing and exploring the suicidal thematic, inquiring about protective and risk factors, and promptly initiating both a psychopharmacological treatment and, where possible, psychological support.
\end{abstract}

Keywords: Suicidal behavior, suicide, suicide attempt, suicidal ideation, neurologic diseases, hopelessness.

\begin{abstract}
"Two historic philosophic forces are engaged in an ongoing territorial fight for the ownership of suicide. Broadly speaking, these two forces can be labeled brain and mind - whether suicide is to be understood primarily in psychiatric terms as a biological disorder in the brain and as a mental disease called depression (and treated with antidepressive medications) or whether it is to be best understood as a psychological drama in the mind related to psychological pain and suffering and best addressed by focusing on the reduction of the person's psychache, treated in terms of the sufferer's own vocabulary. [...].
\end{abstract}

The important thing about philosophy, why it matters, is that it has serious practical everyday consequences. It matters how you conceptualize suicide. Your treatment will certainly be guided by it; your life may depend upon it".

Shneidman, E.S. (2005), pp.209-210, in "Phenomenology of Suicide", M. Pompili Editor, Springer, 2017.

\section{INTRODUCTION}

Neurological diseases expose a higher risk of Suicidal Ideation (SI) and Suicidal Behavior (SB), including Suicide Attempts (SA) and completed suicides [1-3]. They also

*Address correspondence to this author at the Department of Psychiatry, Faculty of Medicine, University of Geneva (UNIGE) Rue Michel Servet 1, 1211 Geneva 4, Switzerland; E-mail: alessandra.costanza@unige.ch represent a prime arena to study the etiopathogenic mechanisms underlying suicidality, appearing emblematic of the heterogeneity and complexity of mutual interrelationships that mark this issue. On the one hand, neurological diseases are characterized by strictly biological impairments that affect cerebral systems investigated at the basis of vulnerability to suicide or result in the need for medical treatments for which suicide has been hypothesized among the possible 
side effects $[1,2]$. On the other hand, they question some subjective experiences of neurological patients, up to the experience of a slip in or near to absurd conditions [4]. The aim of this commentary is not to provide a systemic review of suicidality in neurological diseases, but rather to more narrowly focus on a specific viewpoint: to discuss how neurological diseases influence suicidality by providing a meeting point between biological aspects and those of a more existential nature.

\section{REFLECTIONS REGARDING SUICIDALITY IN NEUROLOGICAL DISEASES AND PATIENTS}

\subsection{From Neurobiology to Hopelessness}

In vivo and post-mortem studies have focused on possible substrates of suicidality at anatomic, anatomo-pathologic, biochemical, genetic, epigenetic, and neuroradiological levels. They revealed morphological and functional lesions in certain regions (prefrontal cortex, insular anterior cortex, amygdala, and hypothalamo-hypophysis-adrenal gland axis), dysfunctions involving neurotransmitters pathways (serotonin, noradrenaline, dopamine, glutamate, and GABA), hormones (cortisol, thyroid hormones, and prolactin) or neurotrophic factors (notably Brain-Derived Neurotrophic Factor, BDNF) [5-8]. Any neurological disease involving these areas and systems could have the potential to increase vulnerability to suicide. To date, however, no "neurobiological marker" has yet been formally and consensually identified [9].

At the same time, some individual representations can take a prominent place in the interiority of a patient's suffering from neurological disease. Among these representations, hopelessness plays a primary role. Historically, it has been suggested that hopelessness is a primary and significantly more important indicator of suicidal intentionality than depression [10]. In the words of patients, hopelessness has also been considered as a facet of psychic pain that touches on an "unbearable", "inescapable", and "inexorable" condition leading patients to consider suicide as a possibility [11]. For some neurological diseases, hopelessness can result from the absence of effective treatments, exposure of facing an unpredictable evolution towards relapse/attack recurrence or slow disease progression, a decrease in physical skills or sense of self-identity, progressive social isolation, perception of becoming useless or feeling stigmatized, and, finally, a slip into an absurd condition [4].

An example of possible drift in a patient's subjectivity is semantic dementia (SD), a variant of Fronto-Temporal Dementia (FTD; other variants of FTD are discussed in section 2.3), for which disturbance of the "future-self" has been described among distinguishing features [12]. The patient cannot imagine reproducing in his future what he was or what he did in his past. Semantic impairment results in loss of meaning of perceptions, words, and concepts. The patient may feel "less human" in a world that he can no longer "describe", "read", and "integrate". Thus, hopelessness may be related to a loss of meaning, especially in the neurological patient who has lost the motor, sensory, and mental capacity to relate to, understand, and belong to that world [4].

\subsection{At the Confluence: The Psychiatric Comorbidity}

Inextricably related to both neurobiological aspects and subjective experiences such as hopelessness, neurological diseases frequently present a psychiatric comorbidity which is pivotal in increasing the suicidal risk. All neurologic patients can present with a co-occurrent or antecedent psychiatric disease (mostly major depression, substance use disorder, psychotic disorder, and borderline personality disorder), which is often unrecognized, banalized, and untreated [13].

Two examples for the clinical relevance of psychiatric comorbidity include Huntington's Disease (HD) and Parkinson's Disease (PD). HD, where a psychiatric constellation of symptoms (affective, psychotic, and behavioral) that are part of the pathognomonic triad associated with chorea-type motor disorders and cognitive disorders progressing to corticalsubcortical dementia, is known to have the highest suicide rate amongst neurological diseases. HD patients are at an increased risk of SI, SA, and completed suicide throughout the entire course of the disease. The most consistent incidence rates, according to a recent systematic review by Kachian et al. [14], are approximately $20 \%$ for SI, $7 \%$ for SA, and $6.6 \%$ for completed suicides. Depression is considered the most consistent risk factor for suicide in these patients. Both patients and subjects who are at risk of developing the disease $(50 \%$ probability of being a carrier of the autosomal dominant gene mutation with complete penetrance and earlier expression in the offspring when a parent is affected) are concerned for SA [14].

Depression in PD is common, with a prevalence of about $50 \%$ and a bimodal distribution showing peaks at early and late stages of the disease [15]. Its occurrence, eventually characterized by atypical features or greater resistance to psychopharmacological treatments, may appear before the clinically evident extra-pyramidal symptomatology [16]. Some mood fluctuations can reproduce the course of motor fluctuations and are thus described as "non-motor" fluctuations (e.g. depressive symptoms during "off" periods). Interestingly, at present, whether the mechanisms of depression in PD are neurobiologically different from those in the general population remains unclear. As in other neurodegenerative diseases, there is not a consensual definition of the limits and the specificity of affective and motivation-related symptoms, such as apathy [17]. According to a number of neuroimaging studies, these symptoms in PD appear related to morphological (such as loss of white matter configuring a "disconnection syndrome") and/or functional variations in some areas belonging to the depression-related neural network, including cortical-subcortical circuits (medial and inferior prefrontal lobes, thalamus, anterior and posterior cingulate, and amygdala-hippocampus complex) and so not only related to basal ganglia dysfunction [17]. These observations include the reduction in serotonin transmission in some of these areas (e.g. the raphe nucleus) that is amended by the antidepressant treatment. The involvement of dopamine transmission deficit and interactions of the latter with other monoamine (serotonin and norepinephrine) turnover, however, complicate the construction of univocal neurobiological models for the pathophysiology of these neuropsychiatric symptoms [17-19]. 
Despite the fa that SA and completed suicide rates in PD are still controversial, being either higher or lower than that of the general population as shown in different studies (for a recent systematic review, see Berardelli et al. [20]), one unequivocal factor is the severity of the concomitant depressive symptomatology including an increase of SI [20,21]. In different studies, death ideation rates have ranged from 21$28.4 \%$ and of SI from $9-14 \%$ [20, 21]. SI has shown to be associated with increased perceived disability, with hopelessness as a risk factor [21]. Increased SB risk in PD has also been associated with iatrogenic psychotic symptoms and impulse dyscontrol. These latter behaviors (e.g. pathologic gambling, hypersexuality, compulsive shopping, binge eating, and compulsive hobbying, described by the patients as "rewarding" behaviors) particularly manifest in relation to the use of the non-ergo dopamine agonists pramipexole and ropinirole, due to their high affinity for dopamine D3 receptors - primary localized in the limbic system - at lower-end therapeutic doses $(\geq 6 \mathrm{mg}$ ropinirole or $\geq 2 \mathrm{mg}$ pramipexole per day) and, in up to one-third of patients, at target doses ( $\geq$ $12 \mathrm{mg}$ ropinirole or $\geq 4.5 \mathrm{mg}$ pramipexole daily) [22]. The risk of these pathological behaviors related to non-ergo dopaminergic drugs, prescribed as mono and first-line therapy in younger patients, increases dramatically when a levodopa therapy is concomitant [22].

Although a firm consensus has not yet been established [20], a possible increase in SB risks in PD after Deep Brain Stimulation (DBS) surgery, targeting most commonly the internal Globus Pallidum (GPi) and the Subthalamic Nucleus (STN), has been signalized. The first description of this phenomenon on a large patients cohort treated for movement disorders was provided by Burkhard et al. [23], who showed a high rate of completed suicide (6/140 patients, $4 \%)$, with 5 cases in the first postoperative year. In the international multi-centric study including the highest number of PD patients who underwent DBS (over 5000 patients out of 55 centers) performed by Voon et al. [24], attempted and completed suicides percentages were $0.90 \%$ and $0.45 \%$, respectively; completed suicide rate in the first postoperative year $(263 / 100000 /$ year, $0.26 \%)$ was significantly higher than the lowest and the highest age-, gender-, and country-adjusted World Health Organization suicide rate, and remained significantly elevated at the fourth post-operative year $(38 / 100$ $000 /$ year, $0.04 \%$ ). Over the last decade, there has been observed a declining trend in SB among DBS patients [25]. However, a very recent study by Giannini et al. [26] on a single-center cohort of over 500 patients treated during 1993-2016 has again called attention to the eventuality of DBS-related SB: attempted and completed suicides percentages were $4.11 \%$ and $0.75 \%$, respectively; the observed suicide rate in the first postoperative year was higher than the expected age- and gender-adjusted French National Observatory on Suicide Risk rate and it remained similar over the second and third postoperative years. A primary hypothesis on putative neurobiological mechanisms explaining suicidality that after DBS targets the undesired diffusion of the current to non-motor, associative or limbic regions to structures adjacent to GPi or STN such as the ventral tegmental area [23]. Another important factor involves the rapid postDBS reduction in dopaminergic treatment, which might lead to a hypodopaminergic state that unmasks the presence of depression in PD patients [20]. The role of the DBS-induced inactivation of heat shock genes Sirtuins (SIRT1-SIRT7, belonging to a family of histone deacetylases involved in the stress response and damage repair) has also been postulated [20]. A variant of genes Sirtuins with reduced neuroprotective properties seems to be associated with SB in bipolar disorders: since they are sensitive to temperature changes, they may be inactivated by DBS electrodes, that induce local changes in brain temperature, and thus promote the appearance of SB [20]. However, although recent parameter/ treatment/genes modifications may play a role in acute affective changes occasionally observed in some cases, it appears less likely in patients in which suicide completion occurred years after DBS surgery [23]. Effects of suicidality could be more consistent with a documented association of depression with DBS emerging in the chronic post-procedure period [20]. It is plausible that refinement of the candidate's selection for DBS as well as a more consistent post-operative follow up could contribute to a decrease in suicidality-related complications in those centers that have the opportunity to rely on a multidisciplinary team composed of a neurologist, psychiatrist, and psychologist [25].

\subsection{Other Suicide Risks due to Some Specific Neurologic Diseases: A Non-Exhaustive Overview}

Currently there is no consensus on the suicidality theme for all the neurological diseases, and the relatively recent studies taking into account the impact of treatments are constantly evolving. Already over two decades ago, Stenager and Stenager [27] highlighted the methodological difficulty of simultaneously comparing results that consider different types of investigation, target populations, control groups, and statistical instruments.

A neurological disease occurring at a young age is often associated with a higher risk of suicide and this is one of the first factors to be considered in the suicidal risk assessment. This is the case for Multiple Sclerosis (MS), where SA risk has been reported to be at least 3 times greater than the general population and completed suicide risk 7.5-14 times greater (for a systematic review, see Pompili et al. [28]). Completed suicide risk is mainly found in subjects under the age of 40 years, males, and affected by the progressive type [28]. A possible increase in severe depression and suicidal risk in MS patients treated with IFN- $\beta$ has also been described [28].

Young age at onset is also a risk factor for suicide in patients with Amyotrophic Lateral Sclerosis (ALS). A large population-based study in Sweden showed suicidal risk 6 times higher in patients with ALS compared to the general population; this risk was significantly higher in the youngest individuals at the time of the first hospitalization and 11 times higher during the first year afterward [29]. Thereafter, the risk decreased but remained significant during the first 3 years [29]. The hypothesis is that, near the diagnosis, the distress reaction is more intense and that the physical resources required to complete suicide are still present [29]. Other factors, such as lack of an etiologic treatment, progressive disability, and caregiver dependence, have been identified in ALS [30]. These arguments are analogous to those emphasized in $\mathrm{HD}$, which at the moment shares an inexorable course with ALS [14, 31]. 
Two FTD subtypes, SD (see section 2.1) and behavioralvariant FTD dementia (bv-FTD), appear to be dementias with high suicidality risk [32]. The risk of completed suicide in bv-FTD has been estimated to be more than twice to that of the general population [33]. bv-FTD often manifests as (or mimics) depressiveness and apathy, but also "loss of sympathy" and indifference about symptoms that can occult to clinicians and caregivers the risk of suicide [32]. Moreover, bvFTD is sometimes combined with ALS, so there may be a synergistic effect with the risk of ALS-related suicide. It is critical to discuss with these patients the presence or absence of suicidal tendencies. There are no specific studies to support this at the moment, and therefore this would be an important issue to elucidate in the future.

Data are heterogeneous for other types of dementia. In the recent past, it was assumed that Alzheimer's Disease (AD) was not associated with a major risk of acting out, except in subjects whose awareness of deficits has not yet dissolved, who have concomitant depressive symptoms, and who do not exhibit apathy [32]. However, a recent systematic review by Serafini et al. [34] has revealed that AD is associated with a $9.7-20 \%$ increased risk of SI and 'wish to die', $7.4 \%$ for SA, and a moderate risk of completed suicide occurring even many years after the diagnosis. Longitudinal studies are needed to clarify specific suicidal characteristics in this population [34].

Increasing evidence suggests that stroke confers a substantial risk of developing SI and dying by suicide, especially among depressed patients. This provides further support for post-stroke depression and suicidality. Young age and female gender confer a higher risk, both for SI and SB (for a systematic review that reports rates of specific studies, see Pompili et al. [35]). Depression, previous mood disorder, prior history of stroke, and cognitive impairment are other factors that contribute to higher risk [35]. Differences have been reported between patients who develop acute-onset suicidal plans and those who have reported delayed-onset plans, with latter occurring more frequently [35]. Stroke associated with language disorders, particularly on the expressive side, has been reported to be associated with a higher risk of suicide (Geriatric Psychiatry Service of Geneva University Hospital, 2008, unpublished observations). The deepening of this empirical observation could suggest an intriguing area of research on suicidality and the persistent but frustrating need to interrelate with the world through "lost" words.

Rather unexpectedly, the literature did not reveal an increased suicidality risk in patients with brain tumors compared to other types of cancer. In contrast, the risk of SI $(7.8 \%)$ is significantly higher in adult survivors of childhood brain tumors, even several years after treatment has been completed [36]. This has been linked to some iatrogenic sequelae (epileptic seizures, physical and neurological limitations), the experience of a very uncertain hope about the future, and possible feelings of "survivor guilt" [36].

Patients with epilepsy present rates of SA and completed suicides higher than those in the general population $[37,38]$. In particular, a meta-analytic investigation performed by Pompili et al. reports that $32.5 \%$ of all deaths of persons with epilepsy are due to suicide and at least $13.5 \%$ of all registered suicides are made by these patients [37]. These higher rates may be independent of the presence of concomitant psychiatric disorders (even if an association between epilepsy and major depression has been consistently shown). The initial notion that suicidality may be prevalent in temporal forms of epilepsy has not been confirmed in the most recent studies. Patients with generalized seizures reported more limitations in daily activities, which were related to emotional problems, compared to patients with other types of seizures. Differently to that reported in ALS and HD, epileptic patients at increased risk of suicide because of higher levels of hopelessness were older than those who had a lower risk of suicide [39]. Interestingly, SAs and recurrent SAs are associated with epilepsy even before epilepsy manifests, strengthening the hypothesis for a common and bidirectional underlying neurobiological etiology with an as-yet-unknown mechanism $[38,40]$. Following an alert from the US Food and Drug Administration in January 2008 [41] that reported a possible association between SB and the use of antiepileptic drugs (AEDs), many studies have explored the subject but have yielded inconsistent findings, particularly in terms of the risk of SB conferred by specific AEDs [42]. Similarly to DBS for PD, it has been suggested that suicidal risk in patients with epilepsy after surgical treatment is higher than in the general population [37].

\subsection{An Unexpected "Burden of Normality"}

In apparent contrast to the experience of inexorability and hopelessness described so far, it has been reported that patients after surgical treatment for both refractory epilepsy and DBS for PD may be at higher risk of depression and suicidality (SB and SI, shown by the above-mentioned studies). First reports of this concerned epileptic patients who successfully underwent antero-temporal resection, and validation was reported in those who underwent extra-temporal resection [43]. In these epileptic patients, it was proposed that unrealistic hopes about surgery were projected [44, 45].

In a similar way, initially just empirically and clinically observed, it was later documented that PD patients who completed suicide after DBS benefited from considerable functional improvement as a result of this procedure, "so that suicide appeared untimely and grossly paradoxical in view of the long-awaited, highly satisfactory motor function restored at the time of their death" [23]. In PD patients, a construct for trying to explain psychosocial adjustment difficulties after DBS, named the "burden of normality", took shape [46, 47]. These patients, accustomed to an illness having three fundamental characteristics (chronic nature, leading disability, and presenting an unexpected chance for a "dramatic cure"), could present obstacles in adapting themselves to a restored normal life. This ill role reversal may perceive to be difficult until no more acceptable, by which patients may lose the identity they tried over many years to rebuild after PD diagnosis. These difficulties also concern relationships with family and the personal environments of patients.

This construct, mostly investigated in epilepsy and PD, could be generalized and considered as a transversal psychological phenomenon which may concern any patient suffering from a severe and disabling disease, including other neurological diseases, who could benefit from a medical procedure which rapidly and drastically changes their health con- 
dition. With regard to suicidality, particular attention should be dedicated to patients that show any of the manifestations of "burden of normality", such as a) psychological distress (including possible "grieving for the illness" or sense of "lost years"); b) psychiatric symptoms (including not only depressive disorders but also mood elevation with mixed features); c) behavioral changes (including excessive activities or shirking behavior with other somatic complaints); and d) interpersonal imbalances (including clumsy repercussions on restructuring family dynamics) [44, 45]. In view of a better long-term and lasting outcome, a personalized and multidisciplinary pre-surgical treatment evaluation was revealed to be more effective if it takes into account the hopes (or expectations) of the patients and their entourage [48].

\subsection{When Action is Less Incisive: A Paradoxical Hopeful Space in the Listening to What is Hard to Listen?}

Considering an inextricable interweaving of "pathology of the brain" and "pathology of the mind" (in accordance with the provocative distinction in the opening quotation from E.S. Shneidman) emerging in neurological diseases can offer a key guide for any condition in which a suicidal risk occurs and obliges to present a humble face against the plethora of hypotheses put forward in the literature. The latter are indispensable but necessarily partial when confronted with such complex, and, at the bottom of the matter, profoundly mysterious phenomena.

The encounter with suicidal neurologic patients also forces the clinician to question their own experience and position on suicide and existence $[49,50,51]$. Is suicide a reasonable or understandable outcome of the hopelessness that is frequently implicit in neurologic diseases? And does this type of reasonableness lead to a certain resistance, resulting in the generalization of this population and banalization of the suicidal risk? Most of the data and reflections reported so far may indicate a highly deterministic conceptualization of the evolution of certain neurological diseases, in which the patient and his clinician may feel impotent and imprisoned [52]. Could one think that having SI is a way for the patient, on the contrary, to control what little he can do? The "simple" expression of this ideation to a clinician who somehow tries to listen to what is hard to listen could in some circumstances create a relational space where this bidirectional and reciprocal act has the possibility to become liberating and, paradoxically, protective as a restorer of a certain form of hope.

\section{CONCLUSION}

Neurological patients are vulnerable to suicidality. They require special attention from clinicians (neurologists and psychiatrists) in terms of openly verbalizing and exploring the suicidal thematic, inquiring about protective and risk factors (including the frequent co-presence or antecedents of psychiatric pathologies), and promptly initiating both a psychopharmacological treatment and, where possible, psychological support.

\section{LIST OF ABBREVIATIONS}

AD

$=$ Alzheimer's Disease

$\begin{array}{ll}\text { AED } & =\text { Anti-Epileptic Drug } \\ \text { ALS } & =\text { Amyotrophic Lateral Sclerosis } \\ \text { bv-FTD } & =\text { Behavioral-Variant Fronto-Temporal Dementia } \\ \text { DBS } & =\text { Deep Brain Stimulation } \\ \text { FTD } & =\text { Fronto-Temporal Dementia } \\ \text { GPi } & =\text { Internal Globus Pallidum } \\ \text { HD } & =\text { Huntington's Disease } \\ \text { MS } & =\text { Multiple Sclerosis } \\ \text { PD } & =\text { Parkinson's Disease } \\ \text { SA } & =\text { Suicide Attempt } \\ \text { SB } & =\text { Suicidal Behavior } \\ \text { SD } & =\text { Semantic Dementia } \\ \text { SI } & =\text { Suicidal Ideation } \\ \text { STN } & =\text { Subthalamic Nucleus }\end{array}$

ETHICS APPROVAL AND CONSENT TO PARTICIPATE

Not applicable.

\section{HUMAN AND ANIMAL RIGHTS}

No Animals/Humans were used for studies that are base of this research.

\section{CONSENT FOR PUBLICATION}

Not applicable.

\section{AVAILABILITY OF DATA AND MATERIALS}

Not applicable.

\section{FUNDING}

None.

\section{CONFLICT OF INTEREST}

The authors declare no conflict of interest, financial or otherwise.

\section{ACKNOWLEDGEMENTS}

Declared none.

\section{REFERENCES}

[1] Arciniegas DB, Anderson CA. Suicide in neurologic illness. Curr Treat Options Neurol 2002; 4(6): 457-68.

http://dx.doi.org/10.1007/s11940-002-0013-5 PMID: 12354372

[2] Coughlin SS, Sher L. Suicidal behavior and neurological illnesses J Depress Anxiety 2013; 9(1)

[3] Eliasen A, Dalhoff KP, Horwitz H. Neurological diseases and risk of suicide attempt: a case-control study. J Neurol 2018; 265(6): 1303-9.

http://dx.doi.org/10.1007/s00415-018-8837-4 PMID: 29564603

Costanza A, Baertschi M, Weber K, Canuto A. [Neurological diseases and suicide: from neurobiology to hopelessness]. Rev Med Suisse 2015; 11(461): 402-5.

PMID: 25895218 
[5] Pompili M, Gibiino S, Innamorati M, et al. Prolactin and thyroid hormone levels are associated with suicide attempts in psychiatric patients. Psychiatry Res 2012; 200(2-3): 389-94. http://dx.doi.org/10.1016/j.psychres.2012.05.010 PMID: 22748186

[6] van Heeringen C, Bijttebier S, Godfrin K. Suicidal brains: a review of functional and structural brain studies in association with suicidal behaviour. Neurosci Biobehav Rev 2011; 35(3): 688-98. http://dx.doi.org/10.1016/j.neubiorev.2010.08.007 PMID: 20826179

[7] van Heeringen K, Mann JJ. The neurobiology of suicide. Lancet Psychiatry 2014; 1(1): 63-72.

http://dx.doi.org/10.1016/S2215-0366(14)70220-2 PMID: 26360403

[8] Serafini G, Pardini M, Pompili M, Girardi P, Amore M. Understanding suicidal behavior: the contribution of recent resting-state fMRI techniques. Front Psychiatry 2016; 7: 69. http://dx.doi.org/10.3389/fpsyt.2016.00069 PMID: 27148097

[9] Costanza A, D'Orta I, Perroud N, et al. Neurobiology of suicide: do biomarkers exist? Int J Legal Med 2014; 128(1): 73-82. http://dx.doi.org/10.1007/s00414-013-0835-6 PMID: 23430141

[10] Beck AT, Steer RA, Kovacs M, Garrison B. Hopelessness and eventual suicide: a 10-year prospective study of patients hospitalized with suicidal ideation. Am J Psychiatry 1985; 142(5): 559-63. http://dx.doi.org/10.1176/ajp.142.5.559 PMID: 3985195

[11] Williams M. Suicide and attempted suicide: Understanding the cry of pain J M Williams. Penguin Books 2001.

[12] Hsiao JJ, Kaiser N, Fong SS, Mendez MF. Suicidal behavior and loss of the future self in semantic dementia. Cogn Behav Neurol 2013; 26(2): 85-92.

http://dx.doi.org/10.1097/WNN.0b013e31829c671d

PMID: 23812172

[13] Rickards H. Depression in neurological disorders: an update. Curr Opin Psychiatry 2006; 19(3): 294-8.

http://dx.doi.org/10.1097/01.yco.0000218601.17722.5b

PMID: 16612216

[14] Kachian ZR, Cohen-Zimerman S, Bega D, Gordon B, Grafman J. Suicidal ideation and behavior in Huntington's disease: Systematic review and recommendations. J Affect Disord 2019; 250: 319-29. http://dx.doi.org/10.1016/j.jad.2019.03.043 PMID: 30875675

[15] Burn DJ. Beyond the iron mask: towards better recognition and treatment of depression associated with Parkinson's disease. Mov Disord 2002; 17(3): 445-54

http://dx.doi.org/10.1002/mds.10114 PMID: 12112190

[16] Leentjens AFG, Van den Akker M, Metsemakers JFM, Lousberg R, Verhey FR. Higher incidence of depression preceding the onset of Parkinson's disease: a register study. Mov Disord 2003; 18(4): 414-8.

http://dx.doi.org/10.1002/mds.10387 PMID: 12671948

[17] Benoit M, Robert PH. Imaging correlates of apathy and depression in Parkinson's disease. J Neurol Sci 2011; 310(1-2): 58-60. http://dx.doi.org/10.1016/j.jns.2011.07.006 PMID: 21831400

[18] Kostić VS, Filippi M. Neuroanatomical correlates of depression and apathy in Parkinson's disease: magnetic resonance imaging studies. J Neurol Sci 2011; 310(1-2): 61-3. http://dx.doi.org/10.1016/j.jns.2011.05.036 PMID: 21684552

[19] Murai T, Müller U, Werheid K, et al. In vivo evidence for differential association of striatal dopamine and midbrain serotonin systems with neuropsychiatric symptoms in Parkinson's disease. J Neuropsychiatry Clin Neurosci 2001; 13(2): 222-8.

http://dx.doi.org/10.1176/jnp.13.2.222 PMID: 11449029

[20] Berardelli I, Belvisi D, Nardella A, et al. Suicide in Parkinson's Disease: A Systematic Review. CNS Neurol Disord Drug Targets 2019; 18(6): 466-77.

http://dx.doi.org/10.2174/1871527318666190703093345 PMID: 31269887

[21] Berardelli I, Belvisi D, Corigliano V, et al. Suicidal ideation, perceived disability, hopelessness and affective temperaments in patients affected by Parkinson's disease. Int J Clin Pract 2018; $73 \mathrm{e} 13287$

http://dx.doi.org/10.1111/ijcp.13287 PMID: 30339296

[22] Hassan A, Bower JH, Kumar N, et al. Dopamine agonist-triggered pathological behaviors: surveillance in the PD clinic reveals high frequencies. Parkinsonism Relat Disord 2011; 17(4): 260-4. http://dx.doi.org/10.1016/j.parkreldis.2011.01.009
PMID: 21310646

[23] Burkhard PR, Vingerhoets FJG, Berney A, Bogousslavsky J, Villemure JG, Ghika J. Suicide after successful deep brain stimulation for movement disorders. Neurology 2004; 63(11): 2170-2. http://dx.doi.org/10.1212/01.WNL.0000145603.48221.B5 PMID: 15596774

[24] Voon V, Krack P, Lang AE, et al. A multicentre study on suicide outcomes following subthalamic stimulation for Parkinson's disease. Brain 2008; 131(Pt 10): 2720-8.

http://dx.doi.org/10.1093/brain/awn214 PMID: 18941146

[25] Pollak P. Deep brain stimulation for Parkinson's disease - patient selection. Handb Clin Neurol 2013; 116: 97-105.

http://dx.doi.org/10.1016/B978-0-444-53497-2.00009-7 PMID: 24112888

[26] Giannini G, Francois M, Lhommée E, et al. Suicide and suicide attempts after subthalamic nucleus stimulation in Parkinson disease. Neurology 2019; 93(1): e97-e105.

http://dx.doi.org/10.1212/WNL.0000000000007665 PMID: 31101738

[27] Stenager EN, Stenager E. Suicide and patients with neurologic diseases. Methodologic problems. Arch Neurol 1992; 49(12): 1296-303

http://dx.doi.org/10.1001/archneur.1992.00530360098025

PMID: 1449409

[28] Pompili M, Forte A, Palermo M, et al. Suicide risk in multiple sclerosis: a systematic review of current literature. J Psychosom Res 2012; 73(6): 411-7.

http://dx.doi.org/10.1016/j.jpsychores.2012.09.011 PMID: 23148807

[29] Fang F, Valdimarsdóttir U, Fürst CJ, et al. Suicide among patients with amyotrophic lateral sclerosis. Brain 2008; 131(Pt 10): 272933.

http://dx.doi.org/10.1093/brain/awn161 PMID: 18669498

[30] Verschueren A, Kianimehr G, Belingher C, et al. Wish to die and reasons for living among patients with amyotrophic lateral sclerosis. Amyotroph Lateral Scler Frontotemporal Degener 2019; 20(12): $68-73$.

http://dx.doi.org/10.1080/21678421.2018.1530265

PMID: 30430868

[31] Bindler L, Travers D, Millet B. Le suicide dans la maladie de Huntington: état actuel des connaissances. Rev Med Suisse 2009; 5(195): 646-8. [Suicide in Huntington's disease: a review]. PMID: 19365916

[32] Haw C, Harwood D, Hawton K. Dementia and suicidal behavior: a review of the literature. Int Psychogeriatr 2009; 21(3): 440-53. http://dx.doi.org/10.1017/S1041610209009065 PMID: 19368760

[33] Alberici A, Cottini E, Cosseddu M, Borroni B, Padovani A. Suicide risk in frontotemporal lobe degeneration: to be considered, to be prevented. Alzheimer Dis Assoc Disord 2012; 26(2): 194-6. http://dx.doi.org/10.1097/WAD.0b013e3182223254 PMID: 21666430

[34] Serafini G, Calcagno P, Lester D, Girardi P, Amore M, Pompili M. Suicide risk in Alzheimer's disease: a systematic review. Curr Alzheimer Res 2016; 13(10): 1083-99.

http://dx.doi.org/10.2174/1567205013666160720112608 PMID: 27449996

[35] Pompili M, Venturini P, Campi S, et al. Do stroke patients have an increased risk of developing suicidal ideation or dying by suicide? An overview of the current literature. CNS Neurosci Ther 2012; 18(9): 711-21.

http://dx.doi.org/10.1111/j.1755-5949.2012.00364.x

PMID: 22943140

[36] Recklitis CJ, Diller LR, Li X, Najita J, Robison LL, Zeltzer L. Suicide ideation in adult survivors of childhood cancer: a report from the Childhood Cancer Survivor Study. J Clin Oncol 2010; 28(4): 655-61.

http://dx.doi.org/10.1200/JCO.2009.22.8635 PMID: 19841325

[37] Pompili M, Girardi P, Tatarelli G, Angeletti G, Tatarelli R. Suicide after surgical treatment in patients with epilepsy: a meta-analytic investigation. Psychol Rep 2006; 98(2): 323-38. http://dx.doi.org/10.2466/pr0.98.2.323-338 PMID: 16796084

[38] Hesdorffer DC, Ishihara L, Webb DJ, Mynepalli L, Galwey NW, Hauser WA. Occurrence and recurrence of attempted suicide among people with epilepsy. JAMA Psychiatry 2016; 73(1): 80-6. 
http://dx.doi.org/10.1001/jamapsychiatry.2015.2516 PMID: 26650853

[39] Pompili M, Serafini G, Innamorati M, et al. Factors associated with hopelessness in epileptic patients. World J Psychiatry 2014; 4(4): 141-9. http://dx.doi.org/10.5498/wjp.v4.i4.141 PMID: 25540729

[40] Pack AM. Epilepsy and Suicidality: What's the Relationship? Epilepsy Curr 2016; 16(4): 236-8.

http://dx.doi.org/10.5698/1535-7511-16.4.236 PMID: 27582659

[41] Food and Drug Administration. Memorandum: Briefing Document for the July 10, 2008 Advisory Committee Meeting to Discuss Antiepileptic Drugs (AEDs) and Suicidality 2008.

http://www.fda.gov/ohrms/dockets/ac/08/briefing/2008-4372b1-01FDA-Katz.pdf

[42] Pompili M, Baldessarini RJ. Epilepsy: Risk of suicidal behavior with antiepileptic drugs. Nat Rev Neurol 2010; 6(12): 651-3. http://dx.doi.org/10.1038/nrneurol.2010.165 PMID: 21131913

[43] Wrench J, Wilson SJ, Bladin PF. Mood disturbance before and after seizure surgery: a comparison of temporal and extratemporal resections. Epilepsia 2004; 45(5): 534-43.

http://dx.doi.org/10.1111/j.0013-9580.2004.48803.x PMID: 15101835

[44] Wilson S, Bladin P, Saling M. The "burden of normality": concepts of adjustment after surgery for seizures. J Neurol Neurosurg Psychiatry $2001 ; 70(5): 649-56$

http://dx.doi.org/10.1136/jnnp.70.5.649 PMID: 11309460

[45] Wilson SJ, Bladin PF, Saling MM. Paradoxical results in the cure of chronic illness: the "burden of normality" as exemplified following seizure surgery. Epilepsy Behav 2004; 5(1): 13-21.

http://dx.doi.org/10.1016/j.yebeh.2003.11.013 PMID: 14751201
[46] Gilbert F. The burden of normality: from 'chronically ill' to 'symptom free'. New ethical challenges for deep brain stimulation postoperative treatment. J Med Ethics 2012; 38(7): 408-12. http://dx.doi.org/10.1136/medethics-2011-100044 PMID: 22431560

[47] Péron J. Neuropsychological care of patients undergoing deep brain stimulation in neurology and psychiatry: Towards an individualized, integrated approach. Rev Neuropsychol 2016; 8(1): 16-24.

[48] Baertschi M, Flores Alves Dos Santos J, Burkhard P, Weber K Canuto A, Favez N. The burden of normality as a model of psychosocial adjustment after deep brain stimulation for Parkinson's disease: A systematic investigation. Neuropsychology 2019; 33(2): 178-94.

http://dx.doi.org/10.1037/neu0000509 PMID: 30667249

[49] Costanza A, Prelati M, Pompili M. The Meaning in Life in Suicidal Patients: The Presence and the Search for Constructs. A Systematic Review. Medicina (Kaunas) 2019; 55(8): 465.

http://dx.doi.org/10.3390/medicina55080465 PMID: 31405240

[50] Costanza A, Baertschi M, Richard-Lepouriel H, et al. 2020; The Presence and the Search Constructs of Meaning in Life in Suicidal Patients Attending a Psychiatric Emergency Department Front Psychiatry 2020; 11(327)

[51] Costanza A, Amerio A, Odone A, et al. Suicide prevention from a public health perspective. What makes life meaningful? The opinion of some suicidal patients. Acta Biomed 2020; 91(3-S): 128-34. PMID: 32275277

[52] Costanza A, Baertschi M, Richard-Lepouriel H, et al. Demoralization and Its Relationship with Depression and Hopelessness in Suicidal Patients Attending an Emergency Department. Int J Environ Res Public Health 2020; 17(7): 2232. http://dx.doi.org/10.3390/ijerph17072232 PMID: 32225017 